\section{Wound botulism caused by Clostridium subterminale after a heroin injection}

\author{
Paris A. Cook, ${ }^{1}$ Aimee Mishler, ${ }^{1}$ \\ Dan Quan, ${ }^{2}$ Ashley Parrish-Garcia ${ }^{2}$ \\ ${ }^{1}$ Department of Pharmacy; ${ }^{2}$ Department \\ of Emergency Medicine, Maricopa \\ Integrated Health System, University of \\ Arizona, Phoenix, AZ, USA
}

\begin{abstract}
Botulism is caused by toxin production from many species of Clostridium, most commonly Clostridium botulinum as well as $C$. baratii and $C$. butyricum. Development of wound botulism is associated with injection drug users but has also been described in traumatic injuries with exposure to soil. A patient presented to the emergency department with a complaint of descending, progressive weakness. He recently reported skin popping with heroin injections. Heptavalent botulinum antitoxin was obtained from the [Center for Disease Control and Prevention (CDC)]. On hospital day seven, the anaerobic wound cultures resulted with growth of Clostridium subterminale.
\end{abstract}

\section{Introduction}

Botulism is an illness caused by toxin production from many species of the Clostridium genus of bacteria, most commonly Clostridium botulinum, $C$. baratii, and $C$. butyricum. These bacteria are anaerobic, spore-forming, Grampositive organisms found naturally in the soil. The different types of botulism include foodborne botulism from improperly canned or stored foods, infant botulism and adult intestinal toxemia from ingestion of botulinum neurotoxin-producing species of Clostridium in infants and adults, respectively, wound botulism, and iatrogenic botulism as a result of cosmetic or therapeutic botulinum toxin overdoses. Wound botulism can occur when botulinum neurotoxin-producing species of Clostridium infect and germinate in wounds and begin producing toxins. Wound botulism presents most similarly to foodborne botulism with descending flaccid paralysis of facial, swallowing, and respiratory muscles, with the exception that it does not typically have accompanying gastrointestinal symptoms. ${ }^{1-3}$ Development of wound botulism is commonly associated with injection drug users but has also been described in traumatic injuries with exposure to soil.

\section{Case Report}

A 33-year-old male had recently presented to another hospital in the area just prior to admission at our hospital for weakness, fatigue and bilateral hip and leg abscesses. At that time he elected to be treated for cellulitis as an outpatient and was prescribed cephalexin $250 \mathrm{mg}$ by mouth four times daily and sulfamethoxazole-trimethoprim 400-80 mg by mouth twice daily for ten days. He then presented to our hospital two days later with a chief complaint of difficulty swallowing and progressive weakness which he states he had for one week. His past medical history included daily heroin use, administered by skin popping or injecting the drug under the skin either intradermally or subcutaneously, and untreated hepatitis C.On presentation to our hospital, the patient was only able to open his eyelids 2$3 \mathrm{~mm}$ wide and had difficulty keeping them open, diplopia, difficulty swallowing and pooling of secretions after 1-2 words, voice changes, difficulty holding his head up and proximal upper extremity weakness. Strength in his upper extremities were $4 / 5$. Initial vital signs included: blood pressure 136/80 $\mathrm{mmHg}$; pulse rate 106 beats/min; respiratory rate 19 breaths/min; oral temperature $36.5^{\circ} \mathrm{C}\left(97.7^{\circ} \mathrm{F}\right)$; and room air pulse oximetry $98 \%$. Five deep cavity abscesses were incised and drained from the patient's hips and thighs, and standard and anaerobic wound cultures were collected. After a few hours, the weakness became more pronounced in the patient's neck and extremities and breathing became very shallow. The decision was made to intubate the patient in the emergency department for airway protection and impending respiratory failure before transferring him to the [medical intensive care unit (ICU)]. A urine drug screen was positive for amphetamines, opiates, and acetone.

The constellation of symptoms seen in the emergency department was suggestive of botulism, with other differential diagnoses including Guillain-Barré Syndrome and myasthenia gravis. $\mathrm{He}$ reported not having eaten canned food that had been bulging and had not traveled outside of the country recently. Therefore, the suspicion was for possible wound botulism. The Maricopa County Department of Public Health was contacted, and receipt of heptavalent botulinum
Correspondence: Aimee Mishler, Department of Pharmacy, Maricopa Medical Center, 2601 East Roosevelt Street, Phoenix, AZ 85008, USA.

Tel.: +1.602.344.5686 - Fax: +1.602.344.5565. E-mail: aimee.mishler@mihs.org

Key words: Botulism; wound botulism; antitoxin; Clostridium; Clostridium subterminale.

Contributions: PC was the main author who completed the first draft of all sections, excluding the abstract. AM was the author who was the primary editor for the abstract, introduction, and discussion sections and was the author who completed the second draft, excluding the abstract, + coordinated all correspondence between authors, was responsible for compiling each portion of the manuscript, and was responsible for formatting and submitting the manuscript. DQ was the final editor for all portions. AP-G was the author who completed the abstract and was the primary editor for the case report section.

Conflicts of interest: the authors declare no conflicts of interests.

Received for publication: 24 February 2018 .

Revision received: 4 June 2018.

Accepted for publication: 14 June 2018.

This work is licensed under a Creative Commons Attribution-NonCommercial 4.0 International License (CC BY-NC 4.0).

CC Copyright P. A. Cook et al., 2018

Licensee PAGEPress, Italy

Infectious Disease Reports 2018; 10:7654

doi:10.4081/idr.2018.7654

antitoxin (A, B, C, D, E, F, G) - BAT $^{\circledR}$ Equine was coordinated directly with the [Centers for Disease Control and Prevention (CDC)]. The antitoxin arrived by freight air transport the next morning on hospital day two and was administered via infusion approximately nineteen hours after presentation to the emergency department. Serum, stool and wound cultures were sent to the CDC for analysis. Wound cultures were also sent to the hospital's lab for analysis. In addition, vancomycin and piperacillin-tazobactam, were started for broad antibiotic coverage including possible [methicillin-resistant Staphylococcus aureus (MRSA)].

In the medical ICU, the patient's weakness plateaued and antibiotics were deescalated to vancomycin and ampicillinsulbactam for continued coverage of his complicated skin-soft tissue infection. After three days in the ICU (hospital day four), a tracheostomy was performed for the expected long recovery time and a PEG 
tube was inserted. The patient was then transitioned to oral antibiotics, amoxicillinclavulanate $875 \mathrm{mg}$ by mouth twice daily and sulfamethoxazole-trimethoprim 800$160 \mathrm{mg}$, two tablets by mouth twice daily for two weeks. On hospital day seven, the anaerobic wound cultures analyzed by the hospital's lab resulted with growth of Clostridium subterminale. On hospital day ten, results from the CDC serum analysis reported toxin A which was identified using MALDI-TOF mass spectrometry, the wound culture analyzed by the CDC did not report botulinum toxin, and the stool was not analyzed.

After a twelve-day hospital stay, the patient was discharged to a skilled nursing facility. He continued to have bilateral ptosis, difficulty swallowing and generalized weakness. He continued to have $4 / 5$ bilateral deltoid muscle strength but $5 / 5$ strength in all other major muscle groups. He had a tracheostomy placed on hospital day 5 and was being weaned off the ventilator at time of discharge.

\section{Discussion and Conclusions}

Wound botulism is caused by the growth of Clostridium bacteria, most commonly Clostridium botulinum, and subsequent toxin production in a wound. Many Clostridia species including: $C$. botulinum, $C$. butyricum, $C$. argentiense, $C$. baratii, $C$. subterminale, $C$. hastiforme, $C$. sporogenes, and $C$. perfringes, have been shown to produce eight different toxins, A, B, C-a, C-b, D, E, F, and G. However, only certain toxin types have been associated with human botulism which include type A, $\mathrm{B}, \mathrm{E}$ and $\mathrm{F}$ toxins with type $\mathrm{A}, \mathrm{B}$ and $\mathrm{E}$ accounting for the majority of cases. $C$. botulinum has been shown to produce all four toxin types. $C$. baratii has been shown to produce type $\mathrm{E}$ toxin and $C$. butyricum has been shown to produce type $\mathrm{F}$ toxin. Type A toxin is most prevalent in the western United States and type B in the eastern United States and Europe. Type E is associated with the consumption of raw marine animals around the world. ${ }^{2}$ These toxins are taken in by presynaptic nerve endings where they irreversibly cleave to soluble N-ethylmaleimide sensitive fusion protein attachment receptor which prevents acetylcholine-containing vesicles from fusing to the cell membrane and releasing the neurotransmitter into the synapse. This results in a classic flaccid paralysis in patients with botulism. ${ }^{2}$

In our particular case, the organism isolated was of unique interest as it is not commonly described as pathogenic nor isolated from wounds. To our knowledge, this is the first case reporting a Clostridium subterminale positive wound culture with type A toxin identified in the serum sample. C. subterminale is a species that is similar to Group IV Clostridia and has been shown to be able to produce typeG toxin, which is not responsible for human illness and to express the gene capable of producing type B toxin, however, has not been shown to actually produce this toxin. ${ }^{4-6}$ One study which focused on Clostridia infections in post-traumatic wounds identified five cases where the growth of $C$. subterminale was found associated with soil contamination. ${ }^{7}$ C. subterminale has been previously reported in immunocompromised patients undergoing chemotherapy, with three bacteremia cases reported. ${ }^{8-10}$ A separate case of bacteremia in an immunocompetent patient was found after an esophageal rupture. ${ }^{11}$ Additionally, there were two cases reported C. subterminale in pleuropulmonary infections, two cases of soft tissue infection, and one case in an open fracture. ${ }^{12-15}$ None of the studies mentioned above provide information regarding neurologic exam findings or toxin identification. Based on these case reports and available literature examining the ability of $C$. subterminale to produce botulinum toxin it is possible that our patient had a co-infection with another Clostrida species that was not identified in the sole wound culture that was obtained.

The CDC has compiled an annual surveillance study since 2001 of all reported cases of botulism and provide data on the number of botulism cases by type, toxins found, and states where the cases occurred. The most recent CDC Botulism Annual Survey in 2016 reported twenty-four cases of confirmed wound botulism (12\% of all botulism cases that year) and an additional two cases of probable wound botulism. Twenty-three $(95.8 \%)$ of the patients with confirmed wound botulism were injection drug users (22 injected black tar heroin and 1 injected methamphetamine). ${ }^{16}$ There has been a steady number of wound botulism cases reported since 2001 with an average of 23 cases per year. The majority of wound botulism cases in 2016 (15 cases) occurred in California, with the next highest amount in New Mexico (3 cases). Other states where cases were reported include Arizona, Colorado, Utah, Texas, New Jersey, and Hawaii with one case each.

A 1998 study published in JAMA looked at twenty-six cases of wound botulism occurring in California from 1994 to 1996 with the intent of finding common risk factors among these cases. They compared these cases with 110 control patients who were enrolled in methadone programs in California and had never had wound botulism. Both groups reported similar rates, $96 \%$ and $97 \%$ respectively, of [black tar heroin (BTH)] use. Their results showed that a significantly higher percentage of patients with wound botulism were injecting BTH by skin popping either subcutaneously or intramuscularly than their control counterparts $(92 \%$ vs $44 \%$, $\mathrm{P}<0.001) .{ }^{17}$ Other studies have continued to look at wound botulism in injection drugs users, especially those using BTH, and have found similar results. ${ }^{18}$

Early recognition of the signs and symptoms of botulism is important. Due to neuroparalytic effects of the toxin produced by Clostridium bacteria, there is a cluster of symptoms typical of patients presenting with botulism. The most classic signs are cranial nerve palsies and descending flaccid paralysis. The eyes are often reported as being affected first with signs of diplopia and ptosis. This is frequently followed by facial paralysis and paralysis of the muscles associated with chewing and swallowing resulting in difficulty swallowing and forming words. Sometimes, after that, a proximal to distal progression of paralysis occurs in the arms and legs. If paralysis continues, the toxin may eventually affect the diaphragm and accessory breathing muscle resulting in respiratory distress or failure. Respiratory failure may occur earlier in the illness due to upper airway collapse or aspiration ${ }^{1,2}$ Often, this results in patients being intubated and a subsequent stay in the ICU. Wound botulism does not frequently involve the gastrointestinal symptoms that would be seen in foodborne botulism. ${ }^{3}$

Due to the type of symptoms present in botulism infections, there are a number of differential diagnoses that need to be considered. Guillain-Barré syndrome is an autoimmune disease that demyelinates neurons and causes an ascending paralysis. However, the Miller Fischer variant of Guillain-Barré syndrome presents with a descending paralysis, closer mimicking that of botulism. Myasthenia gravis is an autoimmune neuromuscular disease that results in weakness of certain muscle groups, sometimes including facial and respiratory muscles. Bilateral or brainstem strokes can also cause similar symptoms such as facial drooping, limb weakness, and breathing difficulties. ${ }^{1,19}$ Ruling out other differential diagnoses is important, however, until botulism can be definitively ruled out, treatment should be considered.

The treatment of botulism includes the administration of botulinum antitoxin. For wound and foodborne botulism, this refers 
specifically to the equine-derived, heptavalent antitoxin that covers types A, B, $\mathrm{C}, \mathrm{D}, \mathrm{E}, \mathrm{F}$, and $\mathrm{G}$ toxins. This antitoxin is supplied by the CDC. Local or state health departments and the CDC are available 24 hours per day 7 days per week for urgent clinical consultations to discuss whether botulism is likely and whether treatment with botulinum antitoxin is warranted. The antitoxin works by binding to and neutralizing any free botulinum toxin and preventing them from binding to nerve endings blocking the development of paralysis. ${ }^{20}$ Administration of the antitoxin does not affect the toxin that is already bound, therefore, it does not reverse any paralysis that is already developed but will prevent further paralysis from forming. There have been no randomized control trials examining equine botulinum antitoxin as a treatment in humans

Early recognition and treatment is very important as benefits of early antitoxin treatment have been shown. One review published in 2003 looked at seven published, confirmed cases of wound botulism in order to identify the timing of antitoxin administration and subsequent outcomes. Two patients received the antitoxin therapy in four days of symptom onset, four patients within eight to fourteen days, and one patient never received antitoxin. The group of four patients who had treatment initiation after four days of symptom onset had longer lengths of hospital stay, were less likely to be ambulatory on discharge, and went to either a nursing home or rehabilitation facility. ${ }^{21} \mathrm{~A}$ second study from 2009 reviewed twentynine cases of wound botulism. The primary objective of this study was to identify factors associated with mechanical ventilation needs, length of ICU stay, length of hospital stay, complications and death. All of the patients were injection drug users who admitted to using heroin via skin popping. The results of this study showed that increasing the time from presentation to antitoxin administration was associated with an increased length of stay in the ICU. ${ }^{22}$ A third study reviewed 249 patients with confirmed botulism who received the equine-derived heptavalent botulinum antitoxin. This study showed a significantly shorter length of hospital stay (15 vs 25 days) and ICU length of stay (10 vs 17 days) for those patients who were treated early ( $\leq 2$ days) verses those who were treated later. $^{23}$

\section{References}

1. Sobel J. Botulism. Clin Infect Dis 2005;41:1167-73.

2. Horowitz BZ. Botulinum toxin. Crit Care Clin 2005;21:825-39.

3. Centers for Disease Control and Prevention. Botulism. Information for Health Professionals. Available from: https://www.cdc.gov/botulism/healthprofessional.html Accessed: April 12, 2017.

4. Hauschild AHW, Dodds KL. Clostridium botulinum: ecology and control in foods. New York: Marcel Dekker; 1993:3-20.

5. Lewis GE Jr, Kulinski SS, Reichard DW, et al. Detection of clostridium botulinum type $\mathrm{g}$ toxin by enzymelinked immunosorbent assay. Appl Environ Microb 1981;42:1018-22.

6. Franciosa G, Ferreira JL, Hatheway CL. Detection of type a, b, and e botulism neurotoxin genes in clostridium botulinum and other clostridium species by PCR: evidence of unexpressed type $\mathrm{B}$ toxin genes in type A toxigenic organisms. J Clin Microbiol 1994;32: 1911-17.

7. Ibnoulkhatib A, Lacroix J, Moine A, et al. Post-traumatic bone and/or joint limb infections due to clostridium spp. Orthop Traumatol Surg Res 2012;98:696-705.

8. Thind SK, Preis JI. Clostridium subterminale septicemia in a patient with esophageal cancer. IDCases 2014;1:47-9.

9. Miyazaki K, Mori T, Takayama N, et al. Clostridium subterminale septicemia in a recipient of allogeneic cord blood transplantation. Intern Med 2003;42:374-5.

10. Haussen DC, Macedo FY, Caperton CV, et al. Clostridium subterminale sepsis in adult acute lymphoblastic leukemia. Leuk Lymph 2011;52:1137-8.

11. Daganou M, Kyriakoudi A, Moraitou $\mathrm{H}$, et al. Clostridium subterminale septicemia in an immunocompetent patient. IDCases 2016;5:43-5.

12. Grobach SL, Thadepalli H. Isolation of clostridium in human infections: evaluation of 114 cases. J Infect Dis 1975;131:S81-5.

13. Gubler JG, Wuest J, Hany A. Pleuropulmonary infection due to Clostridium subterminale. J Infection 1989;19:277-80.

14. Patel SB, Mahler R. Clostridial pleuropulmonary infections: case report and review of the literature. J Infection 1990;21:81-5.

15. Tappe D, Valenza G, Duwe T, et al. Clostridium subterminale infection secondary to an open fracture. Infect Med 2009;26:28-30.

16. Centers for Disease Control and Prevention. Botulism annual summary, 2016. Atlanta, Georgia: US Department of Health and Human Services, CDC, 2017. Available from: https://www.cdc.gov/botulism/pdf/Botu 1ism-2016-SUMMARY-508.pdf Accessed: June 3, 2018.

17. Passaro DJ, Werner SB, McGee J, et al. Wound botulism associated with black tar heroin among injecting drug users. JAMA 1998;279:859-63.

18. Yuan J, Inami G, Mohle-Boetani J, et al. Recurrent wound botulism among injection drug users in california. Clin Infect Dis 2011;52:862-6.

19. Davis LE, King MK. Wound botulism from heroin skin popping. Curr Neurol Neurosci Rep 2008;8:462-8.

20. Lexicomp: online database. Botulism antitoxin, heptavalent. Available from: https://online.lexi.com/lco/action/doc/r etrieve/docid/patch_f/4230963 Accessed: April 20, 2017.

21. Chang GY, Ganguly G. Early antitoxin treatment in wound botulism results in better outcome. Eur Neurol 2003;49:151-3.

22. Offerman SR, Schaefer M, Thundiyil $\mathrm{JG}$, et al. Wound botulism in injection drug users: time to antitoxin correlates with intensive care unit length of stay. West J Emerg Med 2009;10:251-6.

23. Yu AP, Lin NH, Mahon BE, et al. Safety and improved clinical outcomes of patients treated with new equinederived heptavalent botulinum antitoxin. Clin Infect Dis 2017;66:s57s64. 